Homology, Homotopy and Applications, vol.16(1), 2014, pp.313-332

\title{
GLOBAL ORTHOGONAL SPECTRA
}

\author{
ANNA MARIE BOHMANN
}

\author{
(communicated by Michael A. Mandell)
}

\begin{abstract}
For any compact Lie group $G$, there are several wellestablished definitions of a $G$-equivariant spectrum. In this paper, we develop the definition of a global orthogonal spectrum. Loosely speaking, this is a coherent choice of orthogonal $G$-spectrum for each compact Lie group $G$. We use the framework of enriched indexed categories to make this precise. We also consider equivariant $K$-theory and $\operatorname{Spin}^{c}$-cobordism from this perspective, and we show that the Atiyah-Bott-Shapiro orientation extends to the global context.
\end{abstract}

\section{Introduction}

Let $\mathcal{G}$ be the category of compact Lie groups and homomorphisms. For a fixed group $G \in \mathcal{G}$, we may study $G$-spaces using the techniques and methods of $G$-equivariant algebraic topology. Many classical pieces of algebraic topology generalize to the equivariant world, although calculations are significantly more difficult in this context. We propose to generalize to a "global" equivariant world, in which we have compatible $G$-spectra across the entire category of groups. Several classical spectra in topology do generalize naturally to this context, including complex cobordism and complex $K$-theory. "Globalness" should be thought of as a measure of the naturalness of a cohomology theory: since global spectra are equivariant spectra for any compact Lie group, they determine cohomology theories that reflect any available type of symmetry. Furthermore, these cohomology theories vary coherently with the group of symmetry. Hence one can relate spaces or spectra with different group actions, or even consider entire systems of group actions - see [9] for examples.

The idea of global orthogonal spectra was inspired by the paper $[\mathbf{2}]$ in which Greenlees and May introduce the concept of global $\mathcal{I}_{*}$-functors with smash product. The constructions of the present work use a substantially different categorical framework from that of $[\mathbf{2}]$ in order to better fit into modern understandings of point-set level spectra. Schwede independently develops another modern approach to global homotopy theory in [9] using a different categorical framework.

Equivariant orthogonal spectra, as defined and discussed by Mandell and May [7], are one of several good point-set level categories of equivariant spectra. In this paper, we expand the notion of an equivariant orthogonal spectrum given in [7] to a global

Received August 11, 2013, revised February 27, 2014; published on May 29, 2014. 2010 Mathematics Subject Classification: 55P91, 55P42.

Key words and phrases: equivariant homotopy, homotopy, spectra, global.

Article available at http://dx.doi.org/10.4310/HHA.2014.v16.n1.a17

Copyright (C) 2014, International Press. Permission to copy for private use granted. 
version. We focus on the case of genuine $G$-spectra. In the remainder of this section, we recall the definition of an orthogonal $G$-spectrum. In Section 2, we define the categorical structure used in our model of global spectra; in Section 3, we define this model. Section 4 discusses the examples of complex $K$-theory and $\operatorname{Spin}^{c}$-cobordism after a paper of Joachim [4]. Finally, in Sections 5 and 6, we compare some of the categories in our construction to related categories arising in modeling nonequivariant spectra. These comparisons yield two perspectives on equivariant spectra-in the case of a single group, these perspectives have proved a productive technique for equivariant constructions. We review the single group case in Section 5 and extend to the global case in Section 6. In particular, the comparison of Section 6 gives a preliminary relation between our work and that of $[\mathbf{9}]$.

\section{Acknowledgments}

Thanks to Mike Shulman for sharing early stages of his work on enriched indexed categories, and more importantly, for developing this useful categorical framework. The present work had its early beginnings in the author's thesis and she would like to thank Peter May for his thoughts and support, especially during those initial stages. Thanks also to Paul Goerss, John Greenlees, Mike Hill, Kate Ponto, Stefan Schwede, and Brooke Shipley for various enlightening conversations.

\subsection{Basic definitions}

Fix a compact Lie group $G$. Orthogonal $G$-spectra are usually defined in terms of diagrams on a category whose objects are orthogonal representations of $G$ and whose morphisms are linear isometric isomorphisms; see [7, Chapter 2]. Although the category of all $G$-representations is large, it has a small skeleton. We will define orthogonal $G$-spectra in terms of a particularly well-behaved small version of the category of representations; the author thanks Peter May for suggesting this nicely streamlined approach.

Let $\mathcal{I}_{G}$ be the category whose objects are pairs $\left(\mathbb{R}^{n}, \rho: G \rightarrow O(n)\right)$, where $\rho$ is a homomorphism from $G$ to $O(n)$ and thus endows $\mathbb{R}^{n}$ with the structure of a $G$-representation. Morphisms $\left(\mathbb{R}^{m}, \mu\right) \rightarrow\left(\mathbb{R}^{n}, \rho\right)$ are linear isometric isomorphisms $\mathbb{R}^{m} \rightarrow \mathbb{R}^{n}$; hence, the group of automorphisms of $\left(\mathbb{R}^{n}, \rho\right)$ is $O(n)$ and there are no morphisms between representations of different dimensions. This means that $\mathcal{I}_{G}$ is equivalent to the usual category for indexing genuine orthogonal $G$-spectra, and we will sometimes refer to objects of $\mathcal{I}_{G}$ as "representations." Since we do not require morphisms to commute with the homomorphisms $G \rightarrow O(n)$, we have a $G$-action by conjugation on the hom-spaces of $\mathcal{I}_{G}$; concretely, given $f:\left(\mathbb{R}^{m}, \mu\right) \rightarrow\left(\mathbb{R}^{n}, \rho\right)$, an element $g \in G$ acts on $f$ according to the formula

$$
\text { g.f }=\mathbb{R}^{n} \stackrel{\mu\left(g^{-1}\right)}{\longrightarrow} \mathbb{R}^{n} \stackrel{f}{\rightarrow} \mathbb{R}^{n} \stackrel{\rho(g)}{\longrightarrow} \mathbb{R}^{n} .
$$

Thus the maps fixed by this $G$ action are precisely the equivariant maps.

Similarly, define $\mathcal{T}_{o p_{G}}$ to be the category whose objects are based $G$-spaces and whose morphisms are continuous based maps. The category $\mathcal{T}_{o p_{G}}$ is enriched in based $G$-spaces via conjugation. We implicitly add disjoint basepoints to the morphism spaces in $\mathcal{I}_{G}$ so that it is also enriched in based $G$-spaces. We denote the category 
of based $G$-spaces and equivariant maps by $G \mathcal{T}_{o p}$, so both $\mathcal{T}_{o p}$ and $\mathcal{I}_{G}$ are $G \mathcal{T}_{o p}$ enriched categories.

Remark 1.1. We recall the relevant terminology of enriched category theory for discussing these categories. By a $G$-continuous functor between $G \mathcal{T}$ op-enriched categories, we mean a functor preserving the $G$-space structure on hom spaces: that is, for a $G$-continuous functor $X$ and a morphism $f$, we require

$$
g X(f) g^{-1}=X\left(g f g^{-1}\right)
$$

for all $g \in G$. A natural $G$-transformation between functors $X, Y: \mathcal{C} \rightarrow \mathcal{D}$ is a collection of $G$-equivariant maps $\nu: X(C) \rightarrow Y(C)$ that commute with the morphisms in $\mathcal{C}$ :

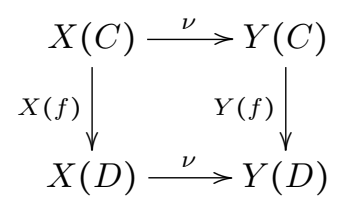

We emphasize that $X(f)$ and $Y(f)$ need not be equivariant maps, but the components $\nu$ must be equivariant.

Notation 1.2. In order to avoid cluttering notation as much as possible, we generally omit explicit mention of the component of a natural transformation such as $\nu$ in our notation. For example, we write $\nu: X(C) \rightarrow Y(C)$ rather than $\nu_{C}$.

Definition 1.3. An $\mathcal{I}_{G}$-space is a $G$-continuous functor $X: \mathcal{I}_{G} \rightarrow \mathcal{T}_{o} p_{G}$. Morphisms

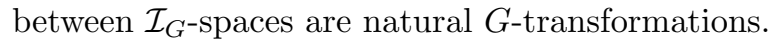

Requiring the morphisms of $\mathcal{I}_{G}$-spaces to be natural $G$-transformations with equivariant components means that the spaces of morphisms between $\mathcal{I}_{G}$-spaces do not have a $G$-action.

Example 1.4. The sphere $\mathcal{I}_{G}$-space $S$ is the functor $V \mapsto S^{V}$ which sends a representation to its one-point compactification.

Example 1.5. Let $V \in \mathcal{I}_{G}$ be an $n$-dimensional $G$-representation, and let

$$
M U(V)=T\left(B U_{n}(V \oplus V)\right)
$$

be the Thom space of the canonical bundle of $n$-planes in $V \oplus V$. The functor $M U_{G}: \mathcal{I}_{G} \rightarrow \mathcal{T}_{o p_{G}}$ given by $V \mapsto M U(V)$ is an $\mathcal{I}_{G}$-space. To get complex cobordism, we should restrict to complex $V$ as in $[\mathbf{2}]$, but as mentioned there and in $[\mathbf{7}$, Section 2.2], this is formally equivalent to a version defined for all $V$.

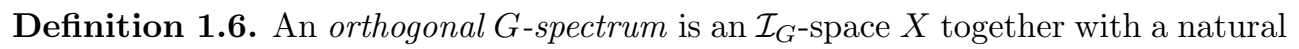
transformation of functors $\mathcal{I}_{G} \times \mathcal{I}_{G} \rightarrow \mathcal{T}_{o p} p_{G}$

$$
X(-) \wedge S^{(-)} \rightarrow X(-\oplus-)
$$

satisfying appropriate associativity and unitality diagrams. In other words, an orthogonal $G$-spectrum is an $\mathcal{I}_{G}$-space with an action of the sphere $\mathcal{I}_{G}$-space. 
Example 1.7. The sphere $\mathcal{I}_{G}$-space $S$ is actually an orthogonal $G$-spectrum, since we have $G$-homeomorphisms $S^{V} \wedge S^{W} \cong S^{V \oplus W}$ for any $G$-representations $V$ and $W$. In fact, $S$ is a strong symmetric monoidal functor from $\mathcal{I}_{G}$ to $\mathcal{T}_{o p}$.

Example 1.8. The functor $M U_{G}$ of Example 1.5 is also an orthogonal $G$-spectrum. The map $M U(V) \wedge S^{W} \rightarrow M U(V \oplus W)$ arises from the Thom construction applied to the map $B U_{n}(V \oplus V) \rightarrow B U_{n+m}(V \oplus W \oplus V \oplus W)$ given by direct sum of an $n$-plane with the basepoint copy of $W$. As in [2], this spectrum is a model for $G$-equivariant complex cobordism. In fact $M U_{G}$ is also a lax symmetric monoidal functor, and thus an orthogonal ring spectrum.

In the sequel, we generalize these definitions to the global case. Heuristically, we wish to have some sort of "fibration" of equivariant spectra over the category $\mathcal{G}$ of compact Lie groups, so that the fiber over any $G \in \mathcal{G}$ is a $G$-spectrum. To make this idea precise, we need to introduce a categorical concept.

\section{2. $\quad$ Enriched indexed categories}

The main difficulty with generalizing the above definition of equivariant orthogonal spectra is understanding how to systematically treat the enrichments. Varying the group of equivariance requires varying the category in which our representations or topological spaces are enriched: there is a natural $G$-structure on maps between $G$ spaces, but a natural $H$-structure on maps between $H$-spaces. Fortunately, Shulman [11] has systematically studied this type of varying enrichment under the name of "enriched indexed categories." He gives two equivalent definitions and we will sketch both of them below.

Intuitively, an enriched indexed category is a fibration of categories with enrichments that vary fiberwise. Here "fibration of categories" should be thought of in the sense of Grothendieck; see Johnstone [5] for a precise definition. In order to make sense of this, we review the standard description of enrichment before moving on to the fiberwise version. Enrichment requires a monoidal category $\mathcal{V}$ to enrich over - in Section 1.1, we used $\mathcal{V}=\mathcal{T}_{o} p_{G}$ when enriching in $G$-spaces. A category $\mathcal{C}$ enriched in $\mathcal{V}$ then has a collection of objects, and for each pair of objects $c, d \in \mathcal{C}$, a "hom-object" $\mathcal{C}(c, d)$ in $\mathcal{V}$ which gives the morphisms from $c$ to $d$. We also require composition and unit data relating the hom-objects.

For the enriched indexed version, we first fix a cartesian monoidal category $S$. This category will be the "base" of the fibrations we are interested in; in other words, $S$ is the category we index over. The monoidal category $\mathcal{V}$ is replaced by a monoidal fibration $\mathcal{V}: V \rightarrow S$ and we refer to enriching in the fibration $\mathcal{V}$. Saying that $\mathcal{V}$ is a monoidal fibration means that $V$ is a monoidal category, $\mathcal{V}$ is a strict monoidal functor, and the monoidal product in $V$ preserves cartesian arrows, which intuitively means that the monoidal product in $V$ preserves the fibrational structure. In our examples, the "base" category of interest is $S=\mathcal{G}$. The monoidal category $V$ is the category $\mathcal{T}_{o p_{\mathcal{G}}}$, whose objects are spaces with an action of some group $G \in \mathcal{G}$ and the monoidal fibration $\mathcal{T}_{o p_{\mathcal{G}}}: \mathcal{T}_{o p_{\mathcal{G}}} \rightarrow \mathcal{G}$ is the functor that sends a space with a group action to the group of equivariance. Thus, we will be concerned with categories indexed on compact Lie groups and enrichments in spaces with actions of the indexing groups. 
Before we can give the first definition of a category enriched in our monoidal fibration $\mathcal{V}$, we need to unpack some of the structure implicit in the fibration. We denote the fiber of $\mathcal{V}$ over an object $X \in S$ by $\mathcal{V}_{X}$; this is a subcategory of $V$. Given a map $f: X \rightarrow Y$ in $S$, the fact that $\mathcal{V}$ is a fibration provides a functor $f^{*}: \mathcal{V}_{Y} \rightarrow \mathcal{V}_{X}$. For any category $\mathcal{C}$ enriched in $\mathcal{V}_{Y}$, we can apply the functor $f^{*}$ to the hom-objects of $\mathcal{C}$ to obtain a category enriched in $\mathcal{V}_{\mathcal{X}}$. We denote the resulting $\mathcal{V}$-enriched category by $\left(f^{*}\right) \mathcal{C}$. If we have a $\mathcal{V}_{Y^{-}}$enriched functor $F: \mathcal{C} \rightarrow \mathcal{D}$, we can also apply $f^{*}$ to obtain a $\mathcal{V}_{X}$ enriched functor $\left(f^{*}\right) \cdot F:\left(f^{*}\right) \cdot \mathcal{C} \rightarrow\left(f^{*}\right) \cdot \mathcal{D}$.

We are now ready to define a $\mathcal{V}$-enriched category. The definition is analogous to defining a fibration of categories as a pseudofunctor from the base category $J$ to the category of categories that picks out the fiber over each $j \in J$.

Definition 2.1 ([11, Definition 4.1]). An indexed $\mathcal{V}$-category $\mathcal{C}$ consists of the following structure.

(a) For each $X \in S$, a category $\mathcal{C}_{X}$ enriched in $\mathcal{V}_{X}$.

(b) For each morphism $f: X \rightarrow Y$ in $S$, a full-and-faithful $\mathcal{V}_{X}$-enriched functor

$$
f^{*}:\left(f^{*}\right) .\left(\mathcal{C}_{Y}\right) \rightarrow \mathcal{C}_{X} \text {. }
$$

(c) For each composable pair of morphisms $X \stackrel{f}{\rightarrow} Y \stackrel{g}{\rightarrow} Z$ in $S$, a $\mathcal{V}_{X}$-natural isomorphism $(g f)^{*} \cong f^{*} \circ\left(f^{*}\right) \bullet g^{*}$.

(d) The isomorphisms of (c) should be compatible with composition in the following sense. For each composable triple $h g f$ of maps in $S$, the following diagram of isomorphisms should commute:

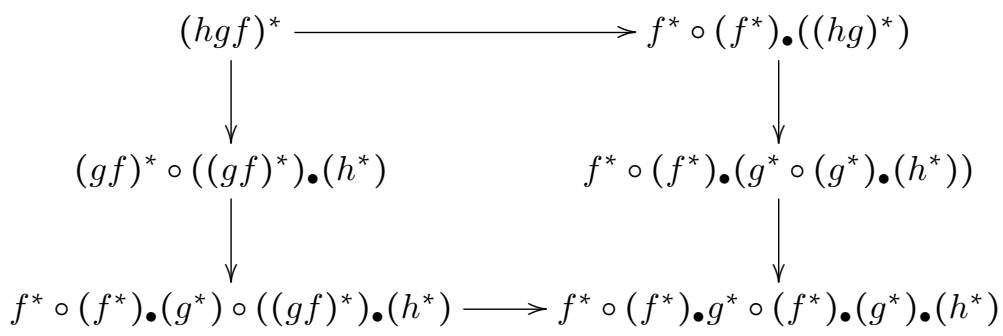

(e) A unit isomorphism $\operatorname{Id}_{\mathcal{C}_{X}} \cong \operatorname{id}_{X}^{*}$ between the identity functor on $\mathcal{C}_{X}$ and the functor induced by the identity map of $X$. Additionally, for $f: X \rightarrow Y$ in $S$, the following diagrams of isomorphisms should commute:
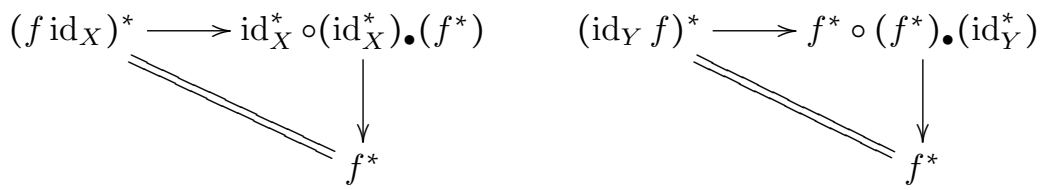

That is, for each indexing object $X \in S$, we have a category enriched in $\mathcal{V}_{X}$. These categories vary coherently in $S$, and the functors relating them preserve as much of the enrichment as possible. This definition is essentially that of a pseudofunctor from $S$ to categories with extra $\left(f^{*}\right)$.'s added when necessary to make enrichments comparable. The categories $\mathcal{I}_{G}$ and $\mathcal{T}_{o p_{G}}$ defined in Section 1 fit together to form indexed $\mathcal{T}_{o p_{\mathcal{G}}}$-categories. 
Example 2.2. $\mathcal{T}_{o} p_{\mathcal{G}}$ is an indexed $\mathcal{T}_{o} p_{\mathcal{G}}$-category in which the $\mathcal{T}_{o} p_{G}$-category corresponding to $G \in \mathcal{G}$ is $\mathcal{T}_{o p_{G}}$ itself. For a homomorphism $\alpha: H \rightarrow G$ in $\mathcal{G}$, the functor $\left(\alpha^{*}\right) \bullet\left(\mathcal{T}_{o} p_{G}\right) \rightarrow \mathcal{T}_{o p} p_{H}$ is given by restriction along $\alpha$. These functors satisfy the required compatibility data.

Example 2.3. Let $\mathcal{I}_{\mathcal{G}}$ denote the indexed $\mathcal{T}_{o} p_{\mathcal{G}}$-category in which the $\mathcal{T}_{o} p_{G}$-category over the index $G \in \mathcal{G}$ is $\left(\mathcal{I}_{\mathcal{G}}\right)_{G}=\mathcal{I}_{G}$. For each homomorphism $\alpha: H \rightarrow G$ in $\mathcal{G}$, the functor $\alpha^{*}:\left(\alpha^{*}\right) .\left(\mathcal{I}_{G}\right) \rightarrow \mathcal{I}_{H}$ is restriction along $\alpha$. This functor sends $\left(\mathbb{R}^{n}, \rho\right) \in \mathcal{I}_{G}$ to $\left(\mathbb{R}^{n}, \rho \circ \alpha\right)$. A direct check shows this satisfies the required compatibility.

We now turn to the second of our equivalent definitions, that of a $\mathcal{V}$-fibration. The underlying basic structure is that of a large $\mathcal{V}$-category. We follow Shulman's notation.

Definition 2.4 ([11, Definition 5.1]). Given a monoidal fibration $\mathcal{V}: V \rightarrow S$, a large $\mathcal{V}$-category $\mathcal{C}$ consists of the following structure.

(a) a collection of objects $x, y, z, \ldots$;

(b) for each $x$, an "extent" $\epsilon x \in S$;

(c) for each pair $x, y$, a hom-object $\underline{\mathcal{C}}(x, y)$ in $\mathcal{V}_{\epsilon x \times \epsilon y}$;

(d) for each $x$, an "identities map" $\mathcal{I}_{\epsilon x} \rightarrow \Delta^{*} \underline{\mathcal{C}}(x, x)$ where $\mathcal{I}_{\epsilon x}$ is the unit of $\mathcal{V}_{\epsilon x}$ and $\Delta: \epsilon x \rightarrow \epsilon x \times \epsilon x$ is the diagonal map;

(e) for each $x, y$ and $z$, a composition map

$$
\underline{\mathcal{C}}(y, z) \otimes_{\epsilon y} \underline{\mathcal{C}}(x, y) \rightarrow \pi_{2}^{*} \underline{\mathcal{C}}(x, z)
$$

where $\pi_{2}$ is the projection from $\epsilon x \times \epsilon y \times \epsilon z$ to $\epsilon x \times \epsilon z$;

(f) composition must be associative and unital in the appropriate sense.

We think of this structure as a category that is in some sense "fibered over" $S$, equipped with a suitable fiber-wise enrichment.

Example 2.5. The category $\mathcal{T}_{o p_{\mathcal{G}}}$ is also a large $\mathcal{T}_{o} p_{\mathcal{G}}$-category. This is the indexed version of viewing $\mathcal{T}_{o} p_{G}$ as a $\mathcal{T}_{o p_{G}}$-enriched category. An object of $\mathcal{T}_{o p_{\mathcal{G}}}$ is a space $X$ with an action by a group $G \in \mathcal{G}$; the group $G$ is its extent. For a $G$-space $X$ and an $H$-space $Y$, the morphism object $\underline{\mathcal{T}}_{\mathcal{G}}(X, Y)=F(X, Y)$ is the $G \times H$-space of based continuous functions $X \rightarrow Y$ where the $G \times H$-action is given by conjugation in the usual way. The identities map is the $G$-equivariant map $S^{0} \rightarrow F(X, X)$ sending the nonbasepoint of the $G$-fixed space $S^{0}$ to the identity map of $X$.

Example 2.6. The category $\mathcal{I}_{\mathcal{G}}$ is also a large $\mathcal{T}_{o} p_{\mathcal{G}}$-category. Here, the objects are pairs $V=\left(\mathbb{R}^{n}, \rho\right) \in \mathcal{I}_{G}$ where $\rho: G \rightarrow O(n)$ for some group $G \in \mathcal{G}$; such a representation has extent $G$. For $V \in \mathcal{I}_{G}$ and $W \in \mathcal{I}_{H}$, the morphism object $\underline{\mathcal{I}}_{\mathcal{G}}(V, W)$ is the $G \times H$ space of linear isometric isomorphisms $V \rightarrow W$ which again has a $G \times H$-action by conjugation. The identities map takes the nonbasepoint of $S^{0}$ to the identity element of $O(n)=\underline{\mathcal{I}}_{\mathcal{G}}(V, V)$.

Definition 2.7 ([11, Definition 6.2]). A $\mathcal{V}$-fibration $\mathcal{C}$ is a large $\mathcal{V}$-category such that for each object $x$ and each morphism $f: Y \rightarrow \epsilon x$ in $S$, there exists a "restriction" $f^{*} x$, 
i.e. an object $f^{*} x$ such that $\epsilon\left(f^{*} x\right)=Y$ and there is a natural isomorphism

$$
\underline{\mathcal{C}}\left(-, f^{*} x\right) \cong(f \times 1)^{*} \underline{\mathcal{C}}(-, x) .
$$

Here "natural" should be understood in a suitable $\mathcal{V}$-categorical sense so that we allow for the change of enrichments when the objects in the blanks vary in extent.

Example 2.8. Both $\mathcal{I}_{\mathcal{G}}$ and $\mathcal{T}_{o p} p_{\mathcal{G}}$ are in fact $\mathcal{T}_{o p_{\mathcal{G}}}$-fibrations. In $\mathcal{T}_{o p_{\mathcal{G}}}$, the restriction of a $G$-space $X$ along a homomorphism $\alpha: H \rightarrow G$ is the $H$-space $\alpha^{*} X$; in $\mathcal{I}_{\mathcal{G}}$, the restriction of a $G$-representation $\left(\mathbb{R}^{n}, \rho\right)$ along $\alpha$ is given by precomposition with $\alpha$. The required natural isomorphism, for example in the case of $\mathcal{T}_{o} p_{\mathcal{G}}$, says that for any space $Z$ with an action of a group $K$, we have an isomorphism of $H \times K$ spaces

$$
F\left(Z, \alpha^{*} X\right) \cong(\alpha \times 1)^{*} F(Z, X)
$$

that is natural in $Z$.

Shulman defines functors and natural transformations of $\mathcal{V}$-fibrations and proves a precise version of the following theorem.

Theorem 2.9 ([11, Theorem 6.7]). The 2-categories of $\mathcal{V}$-fibrations and indexed $\mathcal{V}$ categories are equivalent.

This equivalence is analogous to the equivalence between functors $J \rightarrow \mathcal{C} a t$ and fibrations of categories with base $J$ given by the Grothendieck construction. Given an indexed $\mathcal{V}$-category $\mathcal{C}$, the objects with extent $X$ are the objects of the category $\mathcal{C}_{X}$ indexed by $X$; for $x \in \mathcal{C}_{X}$ and $y \in \mathcal{C}_{Y}$, the hom-object $\underline{\mathcal{C}}(x, y)$ is given by $\mathcal{C}_{X \times Y}\left(\pi_{1}^{*} x, \pi_{2}^{*} y\right)$. In the other direction, if $\mathcal{C}$ is a $\mathcal{V}$-fibration, the corresponding indexed $\mathcal{V}$-category consists of the $\mathcal{V}_{X}$-categories $\mathcal{C}_{X}$ whose objects are the objects with extent $X$ and where morphisms between objects $x$ and $x^{\prime}$ with extent $X$ are given by $\mathcal{C}_{X}\left(x, x^{\prime}\right)=\Delta^{*} \underline{\mathcal{C}}\left(x, x^{\prime}\right)$.

Theorem 2.9 allows us to think of $\mathcal{T}_{o p_{\mathcal{G}}}$ and $\mathcal{I}_{\mathcal{G}}$ both as fibrations over $\mathcal{G}$, with enrichment varying by fiber, and also as collections of indexed categories over each object $G \in \mathcal{G}$, together with appropriate compatibility data for homomorphisms $\alpha \in \mathcal{G}$. In general, our conceptual picture will be based on regarding $\mathcal{T}_{o} p_{\mathcal{G}}$ and $\mathcal{I}_{\mathcal{G}}$ as categories fibered over $\mathcal{G}$, but we will give concrete definitions using their descriptions as indexed $\mathcal{T}_{o p} p_{\mathcal{G}}$-categories.

Shulman also provides a framework for considering monoidal $\mathcal{V}$-fibrations when $\mathcal{V}$ is symmetric monoidal. The 2-category of $\mathcal{V}$-fibrations has a monoidal product $\otimes_{f}$ ( $f$ for "fiberwise") which is given by a fiberwise product of the categories over each extent. Explicitly, if $C$ and $D$ are $\mathcal{V}$-fibrations, then the category of $C \otimes_{f} D$ living over an extent $X$ is the product category $C_{X} \otimes D_{X}$. Hence we have the following definition.

Definition 2.10. A monoidal $\mathcal{V}$-fibration is a monoid in the 2 -category of $\mathcal{V}$-fibrations under $\otimes_{f}$.

Unraveling this definition shows that a monoidal $\mathcal{V}$-fibration is just a $\mathcal{V}$-fibration $\mathcal{C}$ such that each $\mathcal{V}_{X}$-category $\mathcal{C}_{X}$ is monoidal and the transition functors $f^{*}$ together with their coherences are strong monoidal.

Note that one can also define a non-fiberwise monoidal product on $\mathcal{V}$-categories where objects are just pairs of objects, regardless of whether these have the same extent. This product is equivalent to the fiberwise product. 
Theorem 2.11 (Shulman [11, Theorem 11.4]). There is a symmetric monoidal biequivalence between $\mathcal{V}$-fibrations with the fiberwise product and $\mathcal{V}$-categories with this external tensor product.

Hence it suffices to consider the fiberwise tensor product, which is more suitable for our indexed set up.

\section{Defining global spectra}

Our definition of global orthogonal spectra runs essentially parallel to the definition of orthogonal $G$-spectra recalled in Section 1.1, except everything will be fibered over $\mathcal{G}$. To emphasize that $\mathcal{G}$-enrichment has replaced $G$-enrichment, we call our global spectra "orthogonal $\mathcal{G}$-spectra."

Remark 3.1. For categorical reasons, we require that the indexing category be cartesian closed. Throughout, we will use the category $\mathcal{G}$ of all compact Lie groups, but we could easily restrict to a subcategory such as finite groups, Abelian compact Lie groups, or $p$-groups for a fixed prime $p$.

Definition 3.2. An $\mathcal{I}_{\mathcal{G}}$-space is a functor of enriched indexed categories

$$
A: \mathcal{I}_{\mathcal{G}} \rightarrow \mathcal{T}_{o p_{\mathcal{G}}} .
$$

Concretely, the functor $A$ consists of $G$-continuous functors $A_{G}: \mathcal{I}_{G} \rightarrow \mathcal{T}_{o p}$ for all $G \in \mathcal{G}$ such that for each homomorphism $\alpha: G \rightarrow H$, there is a natural isomorphism of $G$-continuous functors filling in the square:

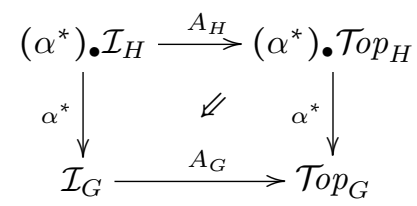

Denote this natural transformation by $\phi_{\alpha}^{A}: \alpha^{*} \circ A_{H} \Rightarrow A_{G} \circ \alpha^{*}$. These natural isomorphisms must be compatible with composition of group homomorphisms, so that $\phi_{\alpha}^{A} \circ \phi_{\beta}^{A}=\phi_{\alpha \beta}^{A}$. They must also satisfy the unit condition $\phi_{\mathrm{id}}=\mathrm{id}$.

Morphisms of $\mathcal{I}_{\mathcal{G}}$-spaces are natural transformations of enriched indexed functors. As such, a morphism $f: A \rightarrow B$ consists of natural $G$-transformations of the form $f_{G}: A_{G} \rightarrow B_{G}$ for each $G \in \mathcal{G}$, and these natural transformations are required to commute with the natural isomorphisms in (1). In other words, we have a commutative diagram of natural transformations

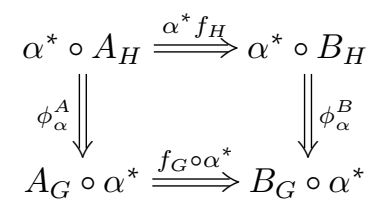

We wish to emphasize that, as $f_{G}$ is a natural $G$-transformation, for each $V \in \mathcal{I}_{G}$, the component $f_{G}: A_{G}(V) \rightarrow B_{G}(V)$ must be a $G$-map. These morphisms make the category $\mathcal{I}_{\mathcal{G}}$-spaces into a topologically enriched category. 
Remark 3.3. Applying the compatibility condition of Equation (1) to the homomorphism $\iota: G \rightarrow e$ yields a natural isomorphism

$$
A_{G} \circ \iota^{*} \cong \iota^{*} A_{e}
$$

which shows that if $V$ is a trivial $G$-representation (so that $V$ is contained in $\iota^{*}\left(\mathcal{I}_{e}\right.$ ) in $\left.\mathcal{I}_{G}\right)$, then $A_{G}(V)$ has a trivial $G$-action. This observation is the essential point to proving an equivalence between $\mathcal{I}_{\mathcal{G}}$-spaces and "I-spaces," which we carry out in Section 6.

Example 3.4. The most fundamental example is the one-point compactification sphere functor $S$, which sends a representation $V$ to its one-point compactification $S^{V}$. If $V \in \mathcal{I}_{G}$ is a $G$-representation and $\alpha: H \rightarrow G$ is a homomorphism, we have a homeomorphism of $H$-spaces $\alpha^{*}\left(S^{V}\right) \cong S^{\alpha^{*} V}$, which is the heart of the required isomorphism (1) of functors from Definition 3.2. We will just call this the sphere functor.

Example 3.5. The complex cobordism functors of Example 1.5 also form an $\mathcal{I}_{\mathcal{G}}$-space. If $V \in \mathcal{I}_{G}$ is a $G$-representation and $\alpha: H \rightarrow G$ is a homomorphism, the required homeomorphism of $H$-spaces $\alpha^{*} M U(V) \rightarrow M U\left(\alpha^{*} V\right)$ is the identity.

We now turn to understanding the monoidal structures involved. Recall from Section 2 that a monoidal $\mathcal{T}_{o p_{\mathcal{G}}}$-fibration is a $\mathcal{T}_{o} p_{\mathcal{G}}$-fibration $\mathcal{C}$ with a monoidal structure on each category $\mathcal{C}_{G}$ and strong monoidal transition functors $\alpha^{*}$. Both $\mathcal{T}_{o p_{\mathcal{G}}}$ itself and $\mathcal{I}_{\mathcal{G}}$ are examples. The smash product of based spaces provides the monoidal structure on each category $\mathcal{T}_{o p_{G}}$ and it is clear that changing the group action via restriction is strongly monoidal. The monoidal structure on $\mathcal{I}_{G}$ is given by direct sum together with the canonical identification $\mathbb{R}^{n} \oplus \mathbb{R}^{m} \cong \mathbb{R}^{n+m}$. More explicitly, $\left(\mathbb{R}^{n}, \rho\right) \oplus\left(\mathbb{R}^{m}, \mu\right)=\left(\mathbb{R}^{n+m}, \rho \oplus \mu\right)$, where $\rho \oplus \mu: G \rightarrow O(n+m)$ is the homomorphism that sends $g \in G$ to the block matrix

$$
\left[\begin{array}{cc}
\rho(g) & 0 \\
0 & \mu(g)
\end{array}\right]
$$

Precomposition with a homomorphism $\alpha: H \rightarrow G$ is strong monoidal: that is,

$$
\alpha^{*}(\rho \oplus \mu)=\alpha^{*} \rho \oplus \alpha^{*} \mu .
$$

The monoidal structure on $\mathcal{I}_{\mathcal{G}}$ allows us to make the following definition.

Definition 3.6. An orthogonal $\mathcal{G}$-spectrum is an $\mathcal{I}_{\mathcal{G}}$-space $A$ together with an associative and unital action of the sphere functor. In other words, there is a natural transformation of $\mathcal{T}_{o p_{\mathcal{G}}}$ functors $\mathcal{I}_{\mathcal{G}} \otimes_{f} \mathcal{I}_{\mathcal{G}} \rightarrow \mathcal{T}_{o p_{\mathcal{G}}}$ whose component at $(V, W) \in \mathcal{I}_{\mathcal{G}} \otimes_{f} \mathcal{I}_{\mathcal{G}}$ is

$$
\sigma_{(V, W)}: A(V) \wedge S^{W} \rightarrow A(V \oplus W) .
$$

This definition uses an external notion of smash product on $\mathcal{I}_{\mathcal{G}}$-spaces. There is an internal smash product given by left Kan extension in the usual way.

Definition 3.7. The (internal) smash product of two $\mathcal{I}_{\mathcal{G}}$-spaces $A$ and $B$ is the left Kan extension of their external smash product along the $\oplus$ functor $\mathcal{I}_{\mathcal{G}} \otimes_{f} \mathcal{I}_{\mathcal{G}} \rightarrow \mathcal{I}_{\mathcal{G}}$. 
Such left Kan extensions exist because the categories $\mathcal{I}_{\mathcal{G}}$ and $\mathcal{T}_{o} p_{\mathcal{G}}$ are both wellbehaved colimit-wise [11, Section 9].

Having monoidal structures on $\mathcal{I}_{\mathcal{G}}$ and $\mathcal{T}_{o} p_{\mathcal{G}}$ allows us to define ring orthogonal $\mathcal{G}$-spectra. If $A: \mathcal{I}_{\mathcal{G}} \rightarrow \mathcal{T}_{o} p_{\mathcal{G}}$ is a lax monoidal functor of $\mathcal{T}_{o} p_{\mathcal{G}}$-fibrations, then the unit $\eta: S \rightarrow A$ and multiplication $\mu: A(-) \wedge A(-) \rightarrow A(-\oplus-)$ give $A$ the structure of an orthogonal $\mathcal{G}$-spectrum via

$$
A(V) \wedge S^{W} \stackrel{\operatorname{id} \wedge \eta}{\longrightarrow} A(V) \wedge A(W) \stackrel{\mu}{\rightarrow} A(V \oplus W) .
$$

Thus any lax monoidal functor $\mathcal{I}_{\mathcal{G}} \rightarrow \mathcal{T}_{o} p_{\mathcal{G}}$ gives a global orthogonal spectrum. Two examples are the sphere spectrum and complex cobordism of Examples 3.4 and 3.5. We will define two more such functors in the next section.

\section{4. $K$-theory and $\operatorname{Spin}^{c}$-cobordism}

For an arbitrary compact Lie group $G$, Michael Joachim constructs $G$-equivariant $K$-theory as an orthogonal $G$-spectrum [4]. He also constructs an orthogonal version of $\operatorname{MPin}_{G}^{c}$ and a map of orthogonal spectra realizing the complex orientation on $K$-theory. We show that these orthogonal spectra for different groups $G$ in fact form an orthogonal $\mathcal{G}$-spectrum.

For each compact Lie group $G$, Joachim constructs an $\mathcal{I}_{G}$-space $\mathbb{K}_{G}$ using $\mathbb{Z} / 2 \mathbb{Z}$ graded $C^{*}$-algebras with $G$ action. He then shows that $\mathbb{K}_{G}$ in fact represents $G$ equivariant complex $K$-theory. Surprisingly, this is the only known $E_{\infty}$-version of equivariant complex $K$-theory when $G$ is a compact Lie group; for finite $G$, the $E_{\infty}$ structure on $K_{G}$ follows from [1, Remark VII.4.4]; see also [3].

Definition 4.1 (Joachim [4]). Let $G$ be a compact Lie group. Given a $G$-representation $V$, let $\mathbb{C} l_{V}$ be the Clifford algebra of $V$ and $\mathcal{K}_{V}$ be the $G$ - $C^{*}$-algebra of compact operators on $L^{2}(V)$. Let $\mathfrak{s}$ be the graded $G$ - $C^{*}$-algebra of continuous functions on $\mathbb{R}$ vanishing at infinity with trivial $G$-action. We then define the $K$-theory orthogonal $G$-spectrum for $G$ to be the lax monoidal functor given by

$$
\mathbb{K}_{G}(V)=\operatorname{Hom}_{C^{*}}\left(\mathfrak{s}, \mathbb{C l}_{V} \otimes \mathcal{K}_{V}\right) .
$$

The multiplication of the lax symmetric monoidal structure arises from a comultiplication $\Delta: \mathfrak{s} \rightarrow \mathfrak{s} \otimes \mathfrak{s} ;$ the unit map $\eta: S \rightarrow \operatorname{Hom}\left(\mathfrak{s}, \mathbb{C} l_{V}, \mathcal{K}_{V}\right)$ is discussed in detail in [4].

The $C^{*}$-algebra $\mathfrak{s}$ is a $\mathbb{Z} / 2 \mathbb{Z}$-graded $C^{*}$-algebra with grading given by the even and odd functions. It admits a unique nontrivial $*$-homomorphism to $\mathbb{C}$ given by evaluation at $0 \in \mathbb{R}$. Moreover, $\mathfrak{s}$ is generated by the two functions $u(t)=\left(1+t^{2}\right)^{-1}$ and $v(t)=t\left(1+t^{2}\right)^{-1}$. Under the identification of $\mathfrak{s} \otimes \mathfrak{s}$ with the $C^{*}$-algebra of functions on $\mathbb{R}^{2}$ vanishing at infinity, the comultiplication on $\mathfrak{s}$ is defined on the generating functions by

$$
\Delta(u)(x, y)=\frac{1}{1+x^{2}+y^{2}} \quad \text { and } \quad \Delta(v)(x, y)=\frac{x+y}{1+x^{2}+y^{2}} .
$$

This comultiplication is both coassociative and cocommutative, and this structure makes Joachim's construction of $\mathbb{K}_{G}$ satisfy the associativity and commutativity required for a lax symmetric monoidal functor. 
Theorem 4.2. The $G$-functors $\mathbb{K}_{G}$ of Definition 4.1 define an orthogonal $\mathcal{G}$-spectrum.

The heart of this statement is in understanding the interplay of $\mathbb{K}_{G}$ with the restriction functors $\alpha^{*}$.

Proof. Given $\alpha: H \rightarrow G$ and a $G$-representation $V \in \mathcal{I}_{G}$, we show that

$$
\mathbb{K}_{H}\left(\alpha^{*} V\right)=\alpha^{*} \mathbb{K}_{G}(V)
$$

for any $\alpha: H \rightarrow G$. This follows from examination of how the equivariance arises in Joachim's construction.

More explicitly, for $V=\left(\mathbb{R}^{n}, \rho\right)$, the action of $G$ on the space

$$
\mathbb{K}_{G}(V)=\operatorname{Hom}_{C^{*}}\left(\mathfrak{s}, \mathbb{C} l_{V} \otimes \mathcal{K}_{V}\right)
$$

is exclusively via the action of $G$ on $\mathbb{C} l_{V} \otimes \mathcal{K}_{V}$, since the $C^{*}$-algebra $\mathfrak{s}$ is fixed. Hence we check that $\alpha^{*}\left(\mathbb{C} l_{V} \otimes \mathcal{K}_{V}\right)$ is equal to $\mathbb{C l}_{\alpha^{*} V} \otimes \mathcal{K}_{\alpha^{*} V}$ as $H$-C $C^{*}$-algebras. As $\alpha^{*}\left(\mathbb{C} l_{V} \otimes \mathcal{K}_{V}\right)=\alpha^{*} \mathbb{C} l_{V} \otimes \alpha^{*} \mathcal{K}_{V}$, we check the equality for the Clifford algebra and compact operator pieces separately. The action on the Clifford algebra $\mathbb{C} l_{V}$ is simply the action of $G$ on $V$ extended to the tensor algebra; this action passes to the quotient $\mathbb{C} l_{V}$ because $G$ acts via the orthogonal group on $\mathbb{R}^{n}$ and thus preserves the standard inner product. Hence the action of $H$ on $\alpha^{*} \mathbb{C} l_{V}$ coincides with the extension of the action of $H$ on $\alpha^{*} V$ to the tensor algebra. Thus $\mathbb{C} l_{\alpha^{*} V}=\alpha^{*} \mathbb{C} l_{V}$ as $H-C^{*}$-algebras.

We next check that $\mathcal{K}_{\alpha^{*} V}=\alpha^{*} \mathcal{K}_{V}$. The $G$ - $C^{*}$-algebra $\mathcal{K}_{V}$ is the $C^{*}$-algebra of compact operators on the completion of the pre-Hilbert space of $L^{2}$ functions vanishing at infinity on $V$. Again, since the $G$-action on $\mathbb{R}^{n}$ preserves the inner product, the $C^{*}$-algebra inherits a $G$-action.

To be concrete, $G$ acts on the space of functions from $V$ to $\mathbb{C}$ by conjugation, which, since $\mathbb{C}$ is $G$-fixed, reduces to $g . f=f\left(g^{-1}(-)\right)$. Because $G$ preserves the inner product on $V$, it preserves norms in $V$ and thus the property of vanishing at infinity. Similarly, the $G$-action preserves the $L^{2}$ norm on the function space: for an $L^{2}$ function $f: V \rightarrow \mathbb{C}$ and an element $g \in G$, we find

$$
\int_{V}\left\langle f\left(g^{-1} v\right), f\left(g^{-1} v\right)\right\rangle=\int_{V}\langle f(v), f(v)\rangle
$$

since $g^{-1}$ acts invertibly on $V$. Thus we have a $G$-action on $L^{2}(V)$, which induces a $G$-action by conjugation on operators from $L^{2}(V)$ to itself. This action preserves compact operators: an operator $T$ is compact if and only if the image of any bounded sequence under $T$ contains a convergent subsequence. If $T$ is such an operator, then $g \circ T \circ g^{-1}$ is also compact. For consider a bounded sequence $\left\{f_{i}\right\}$ of functions in $L^{2}(V)$. Since $G$ preserves the $L^{2}$ norm, the sequence $\left\{g^{-1} \cdot f_{i}\right\}$ is also bounded, so its image $\left\{T\left(g^{-1} \cdot f_{i}\right)\right\}$ contains a convergent subsequence, which is Cauchy. Thus the corresponding subsequence of $\left\{g T\left(g^{-1} \cdot f_{i}\right)\right\}$ is Cauchy and thus converges by completeness.

Hence we see that the $G$-action on $\mathcal{K}_{V}$ is induced by the fact that $G$ acts via orthogonal transformations on $\mathbb{R}^{n}$ and thus preserves the inner product structure. Tracing through this action shows that the $H$-inner product on $\alpha^{*} V$ is simply the restriction of the $G$-inner product to the image of $\alpha$ inside $G$. It is thus apparent that 
$\alpha^{*} \mathcal{K}_{V}=\mathcal{K}_{\alpha^{*} V}$. This shows that $\mathbb{K}$ defines an $\mathcal{I}_{\mathcal{G}}$-space

$$
\mathbb{K}: \mathcal{I}_{\mathcal{G}} \rightarrow \mathcal{T}_{o p_{\mathcal{G}}} \text {. }
$$

It remains to show that $\mathbb{K}$ is in fact an orthogonal $\mathcal{G}$-spectrum. In fact $\mathbb{K}$ is a lax monoidal functor of $\mathcal{T}_{o p_{\mathcal{G}}}$-enriched categories $\mathbb{K}: \mathcal{I}_{\mathcal{G}} \rightarrow \mathcal{T}_{o p_{\mathcal{G}}}$ in the sense of Definition 2.10. Joachim shows that for each fixed $G$, the functor $\mathbb{K}_{G}: \mathcal{I}_{G} \rightarrow \mathcal{T}_{o p}$ is lax monoidal; as mentioned in Section 2, the discussion of monoidal structures in $[\mathbf{1 1}$, Section 11] implies that for our $\mathcal{T}_{o} p_{\mathcal{G}}$ fibrations $\mathcal{I}_{\mathcal{G}}$ and $\mathcal{T}_{o} p_{\mathcal{G}}$, a lax monoidal functor of $\mathcal{T}_{o p_{\mathcal{G}}}$ fibrations is equivalent to a lax monoidal functor $\mathcal{I}_{G} \rightarrow \mathcal{T}_{o p_{G}}$ for each $G$, plus appropriate compatibility for the transformations $\phi$. Since the transformations $\phi^{\mathbb{K}}$ are the identity on the point-set level, we certainly have all the required compatibility. Hence the structure maps of Definition 3.6 are given by composing the unit and multiplication natural transformations of the lax monoidal functor $\mathbb{K}$, as in Equation (3).

We next turn to MSpin ${ }^{c}$. Again, the construction of Joachim [4] generalizes to a global spectrum. Since his construction is still in the framework of $C^{*}$-algebras, we first establish some terminology.

For an inner product space $V \in \mathcal{I}_{G}$, the subgroup $\operatorname{Pin}_{V}^{c} \subset \mathbb{C} l_{V}$ is generated by the unit sphere $S(V) \subset \mathbb{C} l_{V}$ together with the elements of the unit circle $S^{1} \subset \mathbb{C} \subset \mathbb{C} l_{V}$. The $\mathbb{Z} / 2$-grading of $\mathbb{C} l_{V}$ restricts to a grading homomorphism $\xi: P i n_{V}^{c} \rightarrow \mathbb{Z} / 2$ and $\operatorname{Pin}_{V}^{C}$ acts on $\mathbb{C} l_{V}$ via conjugation twisted by $\xi$. That is, an element $v \in \operatorname{Pin}_{V}^{c}$ acts on $w \in \mathbb{C} l_{V}$ by $v \cdot w=\xi(v) v w v^{-1}$. This action restricts to an isometry on $V \subset \mathbb{C} l_{V}$ and thus defines a group homomorphism $\varrho_{V}: \operatorname{Pin}_{V}^{c} \rightarrow O_{V}$ which is known to be surjective.

Let $B\left(\mathbb{C} l_{V} \otimes L^{2}(V)\right)$ be the space of bounded operators on $\mathbb{C} l_{V} \otimes L^{2}(V)$ under the strong $*$-topology. Note that the space of compact operators on $\mathbb{C} l_{V} \otimes L^{2}(V)$ is isomorphic to $\mathbb{C} l_{V} \otimes \mathcal{K}_{V}$. Let $\mathbb{U}_{V} \subset B\left(\mathbb{C} l_{V} \otimes L^{2}(V)\right)$ be the subgroup of unitary operators and scalars, which comes with a grading homomorphism $\xi: \mathbb{U}_{V} \rightarrow \mathbb{Z} / 2$. The group $\mathbb{U}_{V}$ acts on $B\left(\mathbb{C} l_{V} \otimes L^{2}(V)\right)$ via conjugation with twisting by $\xi$, and this action factors through the projective group $\mathbb{P} \mathbb{U}_{V}=\mathbb{U}_{V} / S^{1}$. We construct a map $j_{V}: O_{V} \rightarrow \mathbb{P} \mathbb{U}_{V}$ by associating to each $e \in \operatorname{Pin}_{V}^{c}$ the unitary operator $U_{e} \in B\left(\mathbb{C} l_{V} \otimes L^{2}(V)\right)$ defined by

$$
U_{e}(v \otimes f)=e v \otimes\left(f \circ \varrho_{V}(e)^{-1}\right)
$$

for $v \in \mathbb{C} l_{V}$ and $f \in L^{2}(V)$. This defines a homomorphism $\operatorname{Pin}_{V}^{c} \rightarrow \mathbb{U}_{V}$. The kernel of the homomorphism $\varrho_{V}: \operatorname{Pin}_{V}^{c} \rightarrow O_{V}$ maps to $S^{1} \subset \mathbb{U}_{V}$; the quotient thus gives the homomorphism $j_{V}: O_{V} \rightarrow \mathbb{P} \mathbb{U}_{V}$.

Joachim's model of $M \operatorname{Spin}_{G}^{c}$ is the following.

Definition 4.3 ([4, Definition 6.3]). The equivariant spectrum $M \operatorname{Spin}_{G}^{c}$ is modeled by the lax monoidal functor $\mathbb{M} \operatorname{Spin}_{G}^{c}: \mathcal{I}_{G} \rightarrow \mathcal{T}_{o p}$ which is defined as

$$
\mathbb{M} \operatorname{Spin}_{G}^{c}(V)=\mathbb{P U}_{V+} \wedge_{O_{V}} S^{V} \text {. }
$$

The monoidal structure is induced by the natural maps $\mathbb{U}_{V+} \wedge \mathbb{U}_{W+} \rightarrow \mathbb{U}_{V \oplus W_{+}}$and $S^{V} \wedge S^{W} \rightarrow S^{V \oplus W}$ and the unit is given by

$$
S^{V} \cong O_{V+} \wedge O_{V} S^{V} \stackrel{j_{V} \wedge \text { id }}{\longrightarrow} \mathbb{P} U_{V+} \wedge_{O_{V}} S^{V} .
$$

As $G$ varies, these models $\mathbb{M} \operatorname{Spin}_{G}^{c}$ fit together to form an orthogonal $\mathcal{G}$-spectrum. 
Theorem 4.4. The functors $\mathbb{M} S \operatorname{Sin}_{G}^{c}$ of Definition 4.3 form a lax monoidal functor $I_{\mathcal{G}} \rightarrow \mathcal{T}_{o} p_{\mathcal{G}}$ and thus define an orthogonal $\mathcal{G}$-spectrum.

Proof. As in the proof of Theorem 4.2, the change-of-group functors $\alpha^{*}$ are the identity on the underlying spaces involved. Again, this is because the inner product is assumed to be equivariant, and so the definitions of unitary operators and the like are all respected by the group actions in question. Thus $\operatorname{MSpin}^{c}$ defines an $\mathcal{I}_{\mathcal{G}}$-space, and the lax monoidal structure follows from this structure at each group individually.

Finally, we note that there is a map of lax monoidal $\mathcal{I}_{\mathcal{G}}$-spaces $\mathbb{M} \operatorname{Spin}^{c} \rightarrow \mathbb{K}$ which, for $V \in \mathcal{I}_{G}$, is induced by the map

$$
\tilde{\gamma}_{V}: \mathbb{U}_{V+} \wedge S^{V} \rightarrow \operatorname{Hom}_{\mathbb{C}^{*}}\left(\mathfrak{s}, \mathbb{C} l_{V} \otimes \mathcal{K}_{V}\right)
$$

defined by $\tilde{\gamma}_{V}(U, v)=\xi(U) U \eta_{V}(v) U^{*}$. The unit map $\eta$ of $\mathbb{K}_{G}$ is $O_{V}$-equivariant by construction, so $\tilde{\gamma}_{V}$ induces the desired map

$$
\gamma_{V}: \mathbb{P} U_{V+} \wedge O_{V} S^{V} \rightarrow \operatorname{Hom}\left(\mathfrak{s}, \mathbb{C} l_{V} \otimes \mathcal{K}_{V}\right) .
$$

Note that we are using the identification of $\mathbb{C} l_{V} \otimes \mathcal{K}_{V}$ with compact operators on $\mathbb{C} l_{V} \otimes L^{2}(V)$ in order to define the action of $U \in \mathbb{U}_{V}$ on $\mathbb{C} l_{V} \otimes \mathcal{K}_{V}$. Again, since all the change-of-group functors $\alpha^{*}$ are the identity on underlying spaces, the maps $\gamma_{V}$ determine a natural transformation of functors of $\mathcal{T}_{o} p_{\mathcal{G}}$-indexed categories. As Joachim proves that at each group the natural transformation $\gamma$ is a model for the Atiyah-Bott-Shapiro orientation of complex K-theory [4, Theorem 6.9], we arrive at the following conclusion.

Theorem 4.5. The Atiyah-Bott-Shapiro orientation $M$ Spin $^{c} \rightarrow K$ extends to an orientation of orthogonal $\mathcal{G}$-spectra.

\section{5. $\mathcal{I}_{G}$-spaces and $\mathcal{I}$-Gspaces}

These sections are devoted to showing the equivalence between $\mathcal{I}$-spaces and $\mathcal{I}_{\mathcal{G}^{-}}$ spaces mentioned in Remark 3.3. In this section, we warm up by proving that $\mathcal{I}_{G^{-}}$ spaces are equivalent to $\mathcal{I}$ - $G$ spaces for a single group $G$. This is an orthogonal version of a paper of Shimakawa's [10], which proves that $\Gamma_{G}$-spaces and $\Gamma$ - $G$ spaces are equivalent. A version of the equivalence we prove is shown in [7], but without using the enriched category structure. For a fixed group $G$, this equivalence of categories is a key ingredient in comparing different models of genuine orthogonal $G$-spectra. In Section 6 , we turn to the result for $\mathcal{I}_{\mathcal{G}}$-spaces.

Let $G \mathcal{T}_{o p}$ be the category of based $G$-spaces and based equivariant maps between them; this category is enriched in spaces but not in $G$-spaces. Denote by $\mathcal{I}$ the category $\mathcal{I}_{e}$ corresponding to the trivial group; this has as objects the inner product spaces $\mathbb{R}^{n}$.

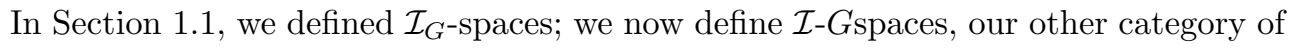
interest.

Definition 5.1. An $\mathcal{I}$-Gspace is a continuous functor $X: \mathcal{I} \rightarrow G \mathcal{T}_{o p}$. Morphisms of $\mathcal{I}$ - $G$ spaces are continuous natural transformations.

Note that the categories of $\mathcal{I}$ - $G$ spaces and $\mathcal{I}_{G}$-spaces are both topologically enriched. 
There is a fully faithful functor $\iota: \mathcal{I} \rightarrow \mathcal{I}_{G}$ given by sending an object $\mathbb{R}^{n} \in \mathcal{I}$ to the object $\left(\mathbb{R}^{n}, \iota\right) \in \mathcal{I}_{G}$, where (by abuse of notation) $\iota$ denotes the unique homomorphism $G \rightarrow O(n)$ that factors through the trivial group. In Section 6, we will have call to think of this functor as precomposition with the unique homomorphism $\iota: G \rightarrow e$. The functor $\iota$ induces a restriction

$$
R: \mathcal{I}_{G^{-}} \text {spaces } \rightarrow \mathcal{I} \text {-Gspaces },
$$

which sends an $\mathcal{I}_{G}$-space $A$ to its restriction to the trivial representations.

Remark 5.2. Any map $f: \mathbb{R}^{m} \rightarrow \mathbb{R}^{n}$ between trivial representations is $G$-fixed, and thus $f$ must be sent to a $G$-fixed map $A(f): A\left(\mathbb{R}^{m}\right) \rightarrow A\left(\mathbb{R}^{n}\right)$. This implies that the restriction of $A$ to the category $\mathcal{I}$ in fact lands in $G \mathcal{T} o p \subset \mathcal{T}$ op $p_{G}$.

In the remainder of this section, we prove the following theorem.

Theorem 5.3. The functor $R: \mathcal{I}_{G}$-spaces $\rightarrow \mathcal{I}$-Gspaces is an equivalence of topological categories.

This is essentially the content of [7, Lemma V.1.1]; our proof is a $G$-enriched version of $[8]$.

This equivalence of categories is at the heart of an important perspective on equivariant homotopy theory. In order to fully capture equivariant structure, a $G$ spectrum is required to contain information about all finite dimensional irreducible representations of $G$. The most straightforward way to do this is by working with the category $\mathcal{I}_{G^{-}}$spaces. Theorem $5.3 \mathrm{implies}$ that this category is equivalent to $\mathcal{I}$-Gspaces. Thus it is common to use the equivalence of Theorem 5.3 to transfer the homotopical information inherent to the $\mathcal{I}_{G}$-spaces framework to $\mathcal{I}$-Gspaces. In essence, this shift in perspective makes the underlying objects of equivariant homotopy simpler, since they are indexed on a smaller category. However, the homotopy theory becomes correspondingly more opaque. Recent work in equivariant homotopy theory has taken advantage of both perspectives.

We now turn to the proof of Theorem 5.3. As is usual, the functor $R$ has a left adjoint "extension" functor $E: \mathcal{I}$-Gspaces $\rightarrow \mathcal{I}_{G}$-spaces given by left Kan extension.

Definition 5.4. For $X \in \mathcal{I}$-Gspaces, define $E X \in \mathcal{I}_{G^{-}}$spaces as the topological left Kan extension $E X=\operatorname{Lan}_{\iota} X$ in the following diagram:

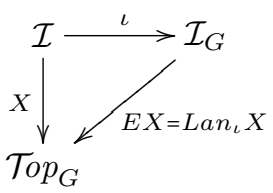

Remark 5.5. As in Remark 5.2, we point out that any functor from $\mathcal{I} \rightarrow \mathcal{T}_{o} p_{G}$ must land in the subcategory $G \mathcal{T} o p$. This legitimizes our regarding $X$ as a functor $\mathcal{I} \rightarrow \mathcal{T}_{o p} p_{G}$ although a priori the left Kan extension only lands in $\mathcal{I} \rightarrow G \mathcal{T} o p$.

For ease of notation, let $V=\left(\mathbb{R}^{n}, \rho\right)$ be an object of $\mathcal{I}_{G}$. Explicitly, the value of $E X$ at $V$ is given by the tensor product of functors

$$
E X(V)=\mathcal{I}_{G}(-, V) \otimes_{\mathcal{I}} X(-)=\mathcal{I}_{G}\left(\mathbb{R}^{n}, V\right) \times_{O(n)} X\left(\mathbb{R}^{n}\right)
$$

where the equivalence relation is given by setting $[s t, x] \sim\left[s, t_{*} x\right]$ for a map $t: \mathbb{R}^{n} \rightarrow \mathbb{R}^{n}$ 
in $O(n)=\mathcal{I}\left(\mathbb{R}^{n}, \mathbb{R}^{n}\right)$. $G$ acts diagonally on $\mathcal{I}_{G}\left(\mathbb{R}^{n}, V\right) \times X\left(\mathbb{R}^{n}\right)$; this action is compatible with the equivalence relation because $t$, as a map between trivial representations, induces an equivariant map $t_{*}$. Furthermore, it is clear that this construction is functorial in $X$, either from the universal property of left Kan extension or via a direct check using the explicit definition.

It is straightforward to check that $E X$ is a $G$-continuous functor. Let $f: V \rightarrow W$ be a map in $\mathcal{I}_{G}$, let $g$ be an element of $G$ and $[s, a] \in E X(V)$. By the definition of the $G$-action on morphisms in $\mathcal{T}_{o p_{G}}$,

$$
\begin{aligned}
(g \cdot E X(f))[s, a] & =g\left(E X(f)\left[g^{-1} s g, g^{-1} a\right]\right) \\
& =g\left(E X(f)\left[g^{-1} s, g^{-1} a\right]\right)
\end{aligned}
$$

since $G$ acts trivially on the source of $s$. Further unraveling of definitions shows

$$
\begin{aligned}
g\left(E X(f)\left[g^{-1} s, g^{-1} a\right]\right) & =\left[g f g^{-1} s g^{-1}, g g^{-1} a\right] \\
& =\left[g f g^{-1} s, a\right]
\end{aligned}
$$

where the second equality again follows from the trivial $G$-action on the source of $s$; hence $g E X(f) g^{-1}=E X\left(g f g^{-1}\right)$.

Since $E$ is defined by left Kan extension, the functors $R$ and $E$ are adjoint. Furthermore, since $\iota: \mathcal{I} \rightarrow \mathcal{I}_{G}$ is the inclusion of a full subcategory, the unit of this adjunction $\operatorname{Id}_{\mathcal{I} \text {-Gspaces }} \rightarrow R E$ is a natural isomorphism by [8, Proposition 3.2]. Thus the following lemma completes the proof of the theorem.

Lemma 5.6. The counit $\epsilon: E R \rightarrow \operatorname{Id}_{\mathcal{I}_{G} \text {-spaces }}$ is a natural isomorphism.

Proof. Let $A: \mathcal{I}_{G} \rightarrow \mathcal{T}_{o p_{G}}$ be an object of $\mathcal{I}_{G}$-spaces. Given a $G$-representation $V=$ $\left(\mathbb{R}^{n}, \rho\right)$, the space $E R A(V)$ is given by

$$
\operatorname{ERA}(V)=\mathcal{I}_{G}\left(\mathbb{R}^{n}, V\right) \times_{O(n)} A\left(\mathbb{R}^{n}\right)
$$

and the $V$ th-component of $\epsilon_{A}$ is given by $[s, a] \mapsto s_{*} a$, where $s_{*}: A\left(\mathbb{R}^{n}\right) \rightarrow A(V)$ is the image of $s \in \mathcal{I}_{G}\left(\mathbb{R}^{n}, V\right)$ under $A$. Note that functoriality of $A$ shows that $\epsilon_{A}$ is well-defined.

For each $A \in \mathcal{I}_{G^{-}}$spaces, $\epsilon_{A}$ must be a $G$-natural transformation; thus each component of $\epsilon_{A}$ must be a $G$-map. This follows from the fact that $A$ preserves the $G$-enrichment, so that $A(g . s)=g . s_{*}$.

We now show $\epsilon_{A}$ is an isomorphism. Suppose $V=\left(\mathbb{R}^{n}, \rho\right)$. Let $\mathbb{R}^{n} \stackrel{i}{\rightarrow} V \stackrel{i^{-1}}{\rightarrow} \mathbb{R}^{n}$ be the (non-equivariant) maps in $\mathcal{I}_{G}$ that are the identity on the underlying vector space $\mathbb{R}^{n}$. We prove that the counit $\epsilon_{A}: E R A(V) \rightarrow A(V)$ is an isomorphism by defining a continuous inverse $\nu$. For $a \in A(V)$, let

$$
\nu_{A}(a)=\left[i, i_{*}^{-1} a\right] .
$$

Continuity of $i_{*}^{-1}$ implies $\nu$ is continuous. A direct check shows that both composites $\epsilon_{A} \circ \nu_{A}$ and $\nu_{A} \circ \epsilon_{A}$ are the identity. Thus $\epsilon$ is a natural isomorphism. Moreover, since $\nu$ provides a point-set inverse to the equivariant map $\epsilon, \nu$ is also equivariant.

We conclude that $R$ and $E$ provide an adjoint equivalence of the categories $\mathcal{I}$-Gspaces and $\mathcal{I}_{G^{-}}$spaces. 


\section{The global version}

We now turn to the global version. This theorem should be thought of as giving an equivalence of categories between the unstable global categories defined in the present paper and in $[\mathbf{9}]$. The basic structure of the proof is the same as in the single group case treated in Section 5, but dealing with the enriched indexed category structure makes the constructions slightly more complicated. We will thus be explicit in making our definitions and checking that they have all the required structure.

The relevant definitions from this paper are in Section 3; in particular the category of $\mathcal{I}_{\mathcal{G}}$-spaces has been defined in Definition 3.2. We now define the other category in our comparison.

Definition 6.1. An $\mathcal{I}$-space is a continuous functor $X: \mathcal{I} \rightarrow \mathcal{T}$ op. Morphisms of $\mathcal{I}$ spaces are continuous natural transformations.

The global analogue of Theorem 5.3 is the following.

Theorem 6.2. The category of $\mathcal{I}_{\mathcal{G}}$-spaces is equivalent to the category of $\mathcal{I}$-spaces. This equivalence is induced by an adjoint pair of functors $R: \mathcal{I}_{\mathcal{G}}$-spaces $\rightarrow \mathcal{I}$-spaces and $E: \mathcal{I}$-spaces $\rightarrow \mathcal{I}_{\mathcal{G}}$-spaces.

Since $\mathcal{I}$-spaces are the structure underlying Schwede's construction of global spec-

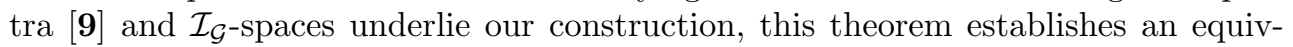
alence between these two structures. Moreover, this theorem should be thought of as establishing two alternative perspectives on global spectra just as Theorem 5.3 establishes two alternative perspectives on $G$-spectra: the perspective of the present work, using $\mathcal{I}_{\mathcal{G}}$-spaces, explicitly incorporates information about all finite dimensional group representations, while Schwede's perspective uses simpler objects and pushes the information about nontrivial representations into the homotopy theory.

Recall that an $\mathcal{I}_{\mathcal{G}}$-space $A$ can be thought of as a collection of suitably compatible functors

$$
A_{G}: \mathcal{I}_{G} \rightarrow \mathcal{T} o p_{G}
$$

In particular, we have a functor $A_{e}: \mathcal{I} \rightarrow \mathcal{T}$ op corresponding to the trivial group $e$. The map $A \mapsto A_{e}$ gives a well-defined forgetful functor $R: \mathcal{I}_{\mathcal{G}}$-spaces $\rightarrow \mathcal{I}$-spaces; this is one of the functors in our equivalence.

The functor $E: \mathcal{I}$-spaces $\rightarrow \mathcal{I}_{\mathcal{G}}$-spaces ultimately comes from the left Kan extensions of Definition 5.4. Let $X: \mathcal{I} \rightarrow \mathcal{T} o p$ be an $\mathcal{I}$-space. For any given compact Lie group $G$, Definition 5.4 provides a functor $E_{G} X: \mathcal{I}_{G} \rightarrow \mathcal{T}_{o} p_{G}$ via left Kan extension. We first must show that these functors fit together to give a well-defined functor of enriched indexed categories $E X: \mathcal{I}_{\mathcal{G}} \rightarrow \mathcal{T}_{o p_{\mathcal{G}}}$; the required enriched indexed functor is then defined by setting $(E X)_{G}=E_{G} X$.

Proposition 6.3. Given an $\mathcal{I}$-space $X$, the functors $E_{G} X$ of Definition 5.4 are the components of an $\mathcal{I}_{\mathcal{G}}$-space.

Proof. We must define the natural isomorphism $G$-functors

$$
\phi_{\alpha}^{E X}: \alpha^{*} \circ E_{H} X \rightarrow E_{G} X \circ \alpha^{*}
$$

corresponding to the homomorphism $\alpha$. Let $V \in \mathcal{I}_{H}$. Because $X\left(\mathbb{R}^{n}\right)$ has trivial group action, we find that $\alpha^{*}\left(E_{H} X(V)\right)=E_{G} X\left(\alpha^{*} V\right)$ as $G$-spaces. It is clear that this 
identification is natural in $V$ and respects composition of group homomorphisms. Hence the collection $\left\{E_{G} X\right\}$ forms an $\mathcal{I}_{\mathcal{G}}$-space.

We now turn to the proof of Theorem 6.2. We begin by defining the unit and counit necessary for the adjunction. The unit has essentially the same structure as the unit of the adjunction from Section 5 .

Definition 6.4. Let $\eta: \operatorname{Id}_{\mathcal{I} \text {-spaces }} \rightarrow R E$ be the natural transformation defined using the natural isomorphism of $\mathcal{I}$-spaces $X \stackrel{\cong}{\rightarrow} R E X=E_{e} X$ that comes from the fact that $\mathcal{I}_{e}=\mathcal{I}$. This is evidently natural in $X$, and provides a natural isomorphism $\eta: \operatorname{Id}_{\mathcal{I} \text {-spaces }} \rightarrow R E$.

Definition 6.5. Let $\epsilon: E R \rightarrow \operatorname{Id}_{\mathcal{I}_{\mathcal{G}} \text {-spaces }}$ be the natural transformation defined as follows. Let $\iota: G \rightarrow e$ be the unique homomorphism. For $A \in \mathcal{I}_{\mathcal{G}}$-spaces, let

$$
\phi_{\iota}^{A}: \iota^{*} A_{e} \rightarrow A_{G} \circ \iota^{*}
$$

be the natural isomorphism of Diagram (1) corresponding to $\iota$. The indexed natural transformation $\epsilon_{A}: E R A \rightarrow A$ has $V$ th component $\epsilon_{A}: E R A(V) \rightarrow A(V)$ defined by

$$
\epsilon_{A}[s, a]=s_{*} \phi_{\iota}^{A}(a)
$$

for $(s, a) \in \mathcal{I}_{G}\left(\mathbb{R}^{n}, V\right) \times A_{e}\left(\mathbb{R}^{n}\right)$.

Lemma 6.6. Definition 6.5 produces a well-defined continuous natural transformation

$$
\epsilon: E R \rightarrow \operatorname{Id}_{\mathcal{I}_{\mathcal{G}} \text {-spaces }} .
$$

Proof. We must check well-definedness at several levels. First, we show that each component of $\epsilon_{A}$ is well defined on the equivalence classes in $E R A(V)$. We must then check that $\epsilon_{A}$ is a morphism of enriched indexed functors. Finally, we show that $\epsilon$ is natural with respect to morphisms of $\mathcal{I}_{\mathcal{G}}$-spaces .

The fact that $\epsilon_{A}$ is well defined on equivalence classes comes from the naturality of $\phi_{\iota}^{A}$. Specifically, consider $t: \mathbb{R}^{n} \rightarrow \mathbb{R}^{n}, s: \mathbb{R}^{n} \rightarrow V$ and $a \in A_{e}\left(\mathbb{R}^{n}\right)$ so that

$$
[s t, a] \sim\left[s, t_{*} a\right] .
$$

Since $\phi_{\iota}^{A}$ is natural with respect to maps of trivial representations,

$$
\begin{aligned}
\epsilon_{A}(s t, a) & =s_{\star} t_{*} \phi_{\iota}^{A} a \\
& =s_{\star} \phi_{\iota}^{A}\left(t_{\star} a\right) \\
& =\epsilon_{A}\left(s, t_{*} a\right)
\end{aligned}
$$

as required.

Next we show that $\epsilon_{A}$ is a morphism in the category $\mathcal{I}_{\mathcal{G}}$-spaces, that is, a natural transformation of enriched indexed functors. For each $G$, the argument of Section 5 shows that our definition of $\epsilon_{A}$ restricts to a natural transformation of continuous $G$-functors $(E R A)_{G}=E_{G} R A \rightarrow A_{G}$. We must show that these natural transformations commute with the compatibility transformations $\phi_{\alpha}^{A}$ and $\phi_{\alpha}^{E R A}$ associated to a 
homomorphism $\alpha: G \rightarrow H$. That is, for each $\alpha: G \rightarrow H$, we must show that we have a commutative diagram of natural transformations

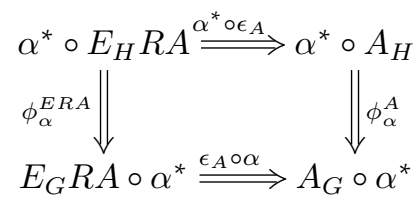

as in Diagram (2).

Consider $V \in \mathcal{I}_{H}$. The map

$$
\phi_{\alpha}^{A} \circ\left(\alpha^{*} \circ \epsilon_{A}\right): \alpha^{*} E_{H} R A(V) \rightarrow A_{G}\left(\alpha^{*} V\right)
$$

sends an element $[s, a]$ to the image of $a \in \alpha^{*} A_{e}\left(\mathbb{R}^{n}\right)$ under the map displayed along the top and right of the following diagram:

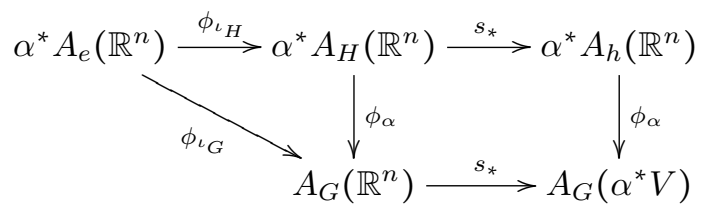

The map

$$
\epsilon_{A} \circ \phi_{\alpha}^{E R A}: \alpha^{*} \circ E_{H} R A(V) \rightarrow A_{G}\left(\alpha^{*} V\right)
$$

is given by sending $(s, a) \in \mathcal{I}_{H}\left(\mathbb{R}^{n}, V\right) \times A_{e}\left(\mathbb{R}^{n}\right)$ to the image of $a$ under the composite along the left and bottom of the same diagram. Commutativity of this diagram follows from naturality of $\phi_{\alpha}$ and the compatibility requirement on the $\phi$ 's: note that $\iota_{G}$ is the composite $\iota_{H} \circ \alpha$.

Lastly, we must show that $\epsilon$ is natural with respect to morphisms in $\mathcal{I}_{\mathcal{G}}$-spaces. Let $f: A \rightarrow B$ be such a morphism. Consider $V \in \mathcal{I}_{G}$. We show that the components of $\left(f \circ \epsilon_{A}\right)$ and $\epsilon_{B} \circ E R f$ at $V$ are equal. Consider the following diagram:

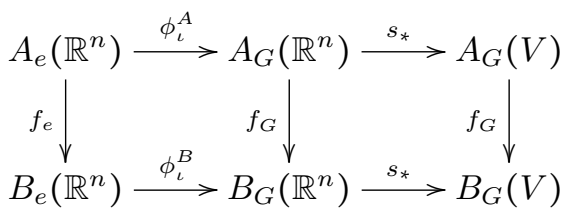

The left square commutes because of the compatibility of $f$ with the natural isomorphisms $\phi^{A}$ and $\phi^{B}$ as in Diagram (2). The right square commutes by naturality of $f_{G}$. If $(s, a) \in \mathcal{I}_{G}\left(\mathbb{R}^{n}, V\right) \times A_{e}\left(\mathbb{R}^{n}\right)$, then $f\left(\epsilon_{A}[s, a]\right)$ is the image of $a$ under the top and right maps in this diagram; $\epsilon_{B}(E R f[s, a])$ is the image of $a$ under the left and lower maps. Thus $\epsilon$ is natural in maps $f: A \rightarrow B$ of $\mathcal{I}_{\mathcal{G}}$-spaces. This completes the proof that $\epsilon$ is a well-defined natural transformation $\epsilon: E R \rightarrow \operatorname{Id}_{\mathcal{I}_{\mathcal{G}} \text {-spaces }}$.

To complete the proof of Theorem 6.2, we must show that $\epsilon$ is a natural isomorphism. We must also show that $\epsilon$ and $\eta$ are adjoint. 
Proof of Theorem 6.2. We define an inverse to $\epsilon$. This definition is nearly the same as that of Equation (5), but we must use the structural natural transformations of Equation (1). For $V=\left(\mathbb{R}^{n}, \rho\right) \in \mathcal{I}_{G}$, let

$$
\mathbb{R}^{n} \stackrel{i}{\rightarrow} V \stackrel{i^{-1}}{\rightarrow} \mathbb{R}^{n}
$$

be the non-equivariant isomorphisms given by forgetting the $G$-action on $V$. For each

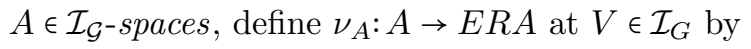

$$
\nu_{A}(a)=\left[i, \phi_{\iota}^{-1} i_{*}^{-1} a\right] .
$$

This map is continuous because $i_{*}^{-1}$ and $\phi_{\iota}^{-1}$ are both continuous.

Consider the composites $\epsilon_{A} \nu_{A}$ and $\nu_{A} \epsilon_{A}$ : for $a \in A_{G}(V)$ and $[s, a] \in E_{G} A(V)$,

$$
\begin{aligned}
\epsilon_{A}\left(\nu_{A}(a)\right) & =i_{\star} \phi_{\iota}\left(\phi_{\iota}^{-1} i_{*}^{-1} a\right) \\
& =a .
\end{aligned}
$$

Naturality of $\phi_{\iota}$ implies $\left(i^{-1} s\right)_{*} \phi_{\iota}=\phi_{\iota}\left(i^{-1} s\right)_{*}$, which provides the third equality in the calculation

$$
\begin{aligned}
\nu_{A}\left(\epsilon_{A}[s, a]\right) & =\left[i, \phi_{\iota}^{-1} i_{\star}^{-1}\left(s_{\star} \phi_{\iota} a\right)\right] \\
& =\left[i, \phi_{\iota}^{-1}\left(i^{-1} s\right)_{\star} \phi_{\iota} a\right] \\
& =\left[i, \phi_{\iota}^{-1} \phi_{\iota}\left(i^{-1} s\right)_{\star} a\right] \\
& =\left[i,\left(i^{-1} s\right)_{\star} a\right] \\
& \sim\left[i i^{-1} s, a\right] \\
& =[s, a] .
\end{aligned}
$$

Thus both composites are the identity; we further need $\nu$ to be an enriched indexed functor. It is standard (see [6, page 16]) to show that component-wise inverses to a natural transformation define an inverse natural transformation; thus, at each $G$, $\nu_{A}: A_{G} \rightarrow E_{G} R A$ is an inverse $G$-natural transformation to $\epsilon_{A}$. Compatibility of $\nu_{A}$ with the natural isomorphisms $\phi$ as in Diagram (2) follows by a similar argument, as does the naturality of $\nu$ with respect to morphisms $A \rightarrow B$ in $\mathcal{I}_{\mathcal{G}}$-spaces. Thus we conclude that $\epsilon: E R \rightarrow \operatorname{Id}_{\mathcal{I}_{\mathcal{G}} \text {-spaces }}$ is a natural isomorphism.

Finally, a direct computation from the definitions shows that $\eta$ and $\epsilon$ satisfy the usual "triangle diagrams" for an adjunction [6, Chapter 5, Theorem 2.v]. Thus $E$ and $R$ define an adjoint equivalence of categories

$$
R: \mathcal{I}_{\mathcal{G}} \text {-spaces } \longleftrightarrow \mathcal{I} \text {-spaces }: E .
$$

Remark 6.7. To maintain consistency with the main body of this paper, we have required the maps in $\mathcal{I}_{G}$ and $\mathcal{I}_{\mathcal{G}}$ to be linear isometric isomorphisms throughout, as is done in defining equivariant orthogonal spectra in [7]. However, all proofs in Sections 5 and 6 work just as well if we allow our maps to merely be linear isometric embeddings. In particular, note that Schwede [9] uses all linear isometries in his definition of "orthogonal space." 


\section{References}

[1] A.D. Elmendorf, I. Kriz, M.A. Mandell and J.P. May, Rings, modules, and algebras in stable homotopy theory, Mathematical Surveys and Monographs, vol. 47, American Mathematical Society, Providence, RI, 1997. With an appendix by M. Cole. MR 1417719 (97h:55006)

[2] J.P.C. Greenlees and J.P. May, Localization and completion theorems for MUmodule spectra, Ann. of Math. (2), 146(3) (1997), 509-544. MR 1491447 (99i:55012)

[3] M.A. Hill and M.J. Hopkins, Equivariant multiplicative closure. 2013. arXiv:math.AT/1303.4479

[4] M. Joachim, Higher coherences for equivariant $K$-theory. In Structured ring spectra, vol. 315 of London Math. Soc. Lecture Note Ser., pages 87-114. Cambridge Univ. Press, Cambridge, 2004. MR 2122155 (2006j:19004)

[5] P.T. Johnstone, Sketches of an elephant: a topos theory compendium. Vol. 1, volume 43 of Oxford Logic Guides. The Clarendon Press Oxford University Press, New York, 2002. MR 1953060 (2003k:18005)

[6] S. Mac Lane, Categories for the working mathematician, vol. 5 of Graduate Texts in Mathematics. Springer-Verlag, New York, second edition, 1998. MR 1712872 (2001j:18001)

[7] M.A. Mandell and J.P. May, Equivariant orthogonal spectra and S-modules, Mem. Amer. Math. Soc., 159 (2002). MR 1922205 (2003i:55012)

[8] M.A. Mandell, J.P. May, S. Schwede and B. Shipley, Model categories of diagram spectra, Proc. London Math. Soc. (3), 82(2) (2001), 441-512. MR 1806878 (2001k:55025)

[9] S. Schwede, Global homotopy theory. 2013. Available on author's webpage.

[10] K. Shimakawa, A note on $\Gamma_{G}$-spaces, Osaka J. Math., 28(2) (1991), 223-228. MR 1132161 (92j:55015)

[11] M. Shulman, Enriched indexed categories, Theory Appl. Categ., 28 (2013), 616-696. MR 3094435

Anna Marie Bohmann bohmann@math.northwestern.edu

Department of Mathematics, Northwestern University, 2033 Sheridan Road, Evanston, IL, 60208, USA 